\title{
In silico screening of 393 mutants facilitates enzyme engineering of amidase activity in CalB
}

Our previously presented method for high throughput computational screening of mutant activity (Hediger et al., PLOS ONE, 2012, 7(12): e49849) is benchmarked against experimentally measured amidase activity for 22 mutants of Candida antarctica lipase B (CalB). Using an appropriate cutoff criterion for the computed barriers, the qualitative activity of 15 out of 22 mutants is correctly predicted. The method identifies four of the six most active mutants with $\geq 3$-fold wild type activity and seven out of the eight least active mutants with $\leq 0.5$-fold wild type activity. The method is further used to screen all sterically possible (386) double-, triple- and quadruple-mutants constructed from the most active single mutants. Based on the benchmark test at least 20 new promising mutants are identified. 


\title{
In silico screening of 393 mutants facilitates enzyme engineering of amidase activity in CalB
}

\author{
Martin R. Hediger ${ }^{1}$, Luca De Vico ${ }^{1}$, Julie B. Rannes ${ }^{2}$, Christian Jäckel ${ }^{2}$, Werner Besenmatter ${ }^{2}$, Allan \\ Svendsen ${ }^{2}$, Jan H. Jensen ${ }^{1 *}$ \\ 1 Department of Chemistry, University of Copenhagen, Universitetsparken 5, DK-2100 \\ Copenhagen, Denmark \\ 2 Novozymes A/S, Krogshoejvej 36, DK-2880 Bagsværd, Denmark \\ * Corresponding Author, Email: jhjensen@chem.ku.dk
}

\begin{abstract}
Our previously presented method for high throughput computational screening of mutant activity (Hediger et al., [1]) is benchmarked against experimentally measured amidase activity for 22 mutants of Candida antarctica lipase B (CalB). Using an appropriate cutoff criterion for the computed barriers, the qualitative activity of 15 out of 22 mutants is correctly predicted. The method identifies four of the six most active mutants with $\geq 3$-fold wild type activity and seven out of the eight least active mutants with $\leq 0.5$-fold wild type activity. The method is further used to screen all sterically possible (386) double-, triple- and quadruple-mutants constructed from the most active single mutants. Based on the benchmark test at least 20 new promising mutants are identified.
\end{abstract}

\section{Introduction}

In industry, one frequently tries to modify an enzyme in order to enhance its functionality in a certain way [2-6]. From an application point of view, one of the most interesting questions is how to modify an enzyme such that its activity is enhanced compared to wild type or such that a new kind of activity is introduced into the enzyme $[7,8]$. It can therefore be of considerable relevance to have a method available which efficiently allows to a priori discriminate between promising candidates for experimental study and mutants which can be excluded from the study. 
Numerous methods are currently being proposed and developed for the description of enzyme activities, the theoretical background of which ranges from phenomenological and bioinformatics based approaches [9-13] to quantum mechanics based ab initio descriptions [14-22]. However one can expect that methods which are highly demanding in terms of set-up efforts and computational time are less likely to be employed in industrial contexts where qualitative or semi-quantitative conclusions can be of sufficient use in the beginning and planning phase of a wet-lab study. Few approaches, while taking into account a number of approximations and limitations in accuracy, aim at being used in parallel or prior to experimental work $[23,24]$ and are not designed to be used for high throughput fashion.

Hediger et al. have recently published a computational method for high throughput computational screening of mutant activity [1] and in this paper we benchmark the method against experimentally measured amidase activity for mutants of Candida antarctica lipase B (CalB) and apply the method to identify additional promising mutants.

\section{Methods}

We introduce the experimental set-up and the methodology for comparing experimental and computational data. We describe a benchmarking and a combinatorial study of CalB mutant activity.

Experimentally, variants of Candida Antarctica lipase B (CalB) were either produced in Pichia pastoris with C-terminal His6-tag for subsequent affinity purification or expressed in Aspergillus oryzae without terminal tag followed by a three-step purification procedure.

It is generally accepted that in serine protease like enzymes, the formation of the tetrahedral intermediate (TI, Fig. 1) is rate determining [25-28] and throughout this work we assume that a lower barrier for this reaction correlates to increased overall activity of the enzyme.

The substrate used throughout this study is N-benzyl-2-chloroacetamide. The organisms used for expression of the individual variants are indicated in Table 1.

\section{Generation of CalB Variants without His-tags}

Variants of CalB carrying the CalB signal peptide were generated at the DNA level using QuickChange mutagenesis on the corresponding gene residing in a dual E.coli/Aspergillus Pichia pastoris expression vector. The PCR was performed with proofreading DNA polymerase (New England Biolabs, NEB). To 
remove parent templates, they were methylated in vitro prior to PCR with CpG methyltransferase (from NEB) and digested in vivo after transformation of competent E.coli DH5 $\alpha$ cells (TaKaRa) according to the instructions from the manufacturer. Plasmid DNA was isolated from transformed E.coli strains, and sequenced to verify the presence of the desired substitutions. Confirmed plasmid variants were used to transform an Aspergillus oryzae strain that is negative in pyrG (orotidine-5'-phosphate decarboxylase), proteases pepC (aserine protease homologous to yscB), alp (an alkaline protease) NpI (a neutral metalloprotease I) to avoid degradation of the lipase variants during and after fermentation.

The transformed Aspergillus strains were fermented as submerged culture in shake flasks and the lipase variants secreted into the fermentation medium. After the fermentation, the lipase variants were purified from the sterile filtered fermentation medium in a 3 step procedure with 1) hydrophobic interaction chromatography on decylamine-agarose, 2) buffer exchange by gel filtration and 3) ion exchange chromatography with cation exchange on SP-sepharose at $\mathrm{pH}$ 4.5. The lipase variant solutions were stored frozen.

\section{Generation of CalB Variants with His-tags}

Variants of CalB carrying the CalB signal peptide and C-terminal His-tags were generated at the DNA level using SOE-PCR and inserted into a dual E.coli/Pichia pastoris expression vector using In-fusion cloning (ClonTech). The SOE-PCR was performed with Phusion DNA polymerase (NEB) and template DNA of the CalB gene. The cloned plasmids were transformed in competent E.coli DH5 $\alpha$ cells (TaKaRa). Plasmid DNA was isolated from transformed E.coli strains, and sequenced to verify the presence of the desired substitutions. Confirmed plasmid variants were used to transform a Pichia pastoris strain that is Mut(s), Suc(+), His(-). The transformed Pichia strains were fermented as submerged culture in deep well plates and secretion of the lipase variants into the fermentation medium was induced by addition of methanol. After the fermentation, the lipase variants were purified from the cleared supernants using a standard His-tag purification protocol (Qiagen) and buffer-exhanged into $50 \mathrm{mM}$ phosphate buffer, $\mathrm{pH}$ 7.0, using Amicon Ultra centrifugal filter devices with a $10 \mathrm{kDa}$ cutoff (Merck Millipore).

\section{Activity Measurement}

Amidase activity of CalB variants was determined in a two-step fluorimetric assay previously described by Henke et al [29]. First, enzymatic hydrolysis of N-benzyl-2-chloroacetamide was performed in 96-well microtiter plates in $200 \mu \mathrm{L}$ phosphate-buffered aqueous solution $\mathrm{pH} 7.0$ including $10 \%$ organic co-solvent 
(THF or DMSO). Reactions containing $5 \mathrm{mM}$ amide substrate, $0.3-3 \mu \mathrm{M}$ enzyme, and $12 \mu \mathrm{g} / \mathrm{mL}$ BSA were incubated for $18-20 \mathrm{~h}$ at $37^{\circ} \mathrm{C}$ in a shaker incubator. In a second step, $50 \mu \mathrm{L}$ of a $20 \mathrm{mM} 4$ nitro-7-chloro-benzo-2-oxa-1,3-diazole (NBD-Cl) solution in 1-hexanol was added and the reaction of NBD-Cl with benzylamine formed during amide hydrolysis proceeded under identical reaction conditions for another hour.

Fluorescence of the final reaction product was determined with excitation at $485 \mathrm{~nm}$ and measured emission at $538 \mathrm{~nm}$. Calibration of the amide hydrolysis reaction was performed on each assay plate with benzylamine covering a concentration range between 0.05 and $5 \mathrm{mM}$. All enzymatic activities were corrected for non-enzymatic background reaction determined under identical conditions without enzyme present.

\section{Computational Details}

The computational method used to estimate the reaction barriers of the CalB mutants has been described in detail earlier [1] and is only summarized here.

As described previously [1], in order to make the method computationally feasible, relatively approximate treatments of the wave function, structural model, dynamics and reaction path are used. Given this and the automated setup of calculations, some inaccurate results will be unavoidable. However, the intent of the method is similar to experimental high throughput screens of enzyme activity where, for example, negative results may result from issues unrelated to intrinsic activity of the enzyme such as imperfections in the activity assay, low expression yield, protein aggregation, etc. Just like its experimental counterpart our technique is intended to identify potentially interesting mutants for further study.

The reaction barriers are estimated computationally by preparing molecular model structures [1] (consisting of around 840 atoms) of the enzyme substrate complex (ES) and the tetrahedral intermediate (TI) inbetween which linear interpolation is carried out to generate structures of the enzyme on the reaction path. Such adiabatic mapping is the most common way to estimate barriers in QM/MM studies of enzymatic reaction mechanisms. The resulting barriers tend to be in good agreement with experiment, which indicates that this is a reasonable approximation (see for example [30] and [16]). The geometry of each interpolation frame is optimized while keeping the distance between the nucleophilic carbon $\mathrm{C}^{20}$ of the substrate and $\mathrm{O}^{\gamma}$ of serine 105 (Fig. 1) fixed at a specific value $d_{i}=d_{i n i}-i\left(d_{i n i}-d_{f i n}\right) / 10$, where $d_{i n i}$ and $d_{f i n}$ are the distances between $\mathrm{C}^{20}$ and $\mathrm{O}^{\gamma}$ in the ES complex and TI, respectively (in $\AA, 10$ 
being the number of interpolation frames and $i$ the interpolation frame index). In geometry optimization calculations, the gradient convergence criteria is set to $0.5 \mathrm{kcal} /(\mathrm{mol} \AA)$ and a linear scaling implementation of the PM6 method (MOZYME [31]) together with a NDDO cutoff of $15 \AA$ is applied. The energy profile of the reaction barrier at the PM6 level of theory [32] is subsequently mapped out by carrying out conventional SCF calculations of each optimized interpolation frame. All calculations are carried out using the MOPAC suite of programs [33,34]. The molecular models are based on the crystal structure of the CalB enzyme with PDB identifier 1LBS [35]. In order to prevent significant rearrangement of hydrogen bonding network of surface residues during the optimization, a number of additional structural constraints are applied in the geometry optimizations, i.e. the residues S50, P133, Q156, L277 and P280 are kept fixed. These (surface) residues are observed to rearrange and form new hydrogen bonds in optimizations when no constraints are applied. Omitting the constraints leads to unconclusive barrier shapes containing many irregular minima along the reaction coordinate which do not permit to readily define a reaction barrier.

For the analysis, the reaction barrier is defined by the difference between the highest energy point on the reaction profile and the energy corresponding to the enzyme substrate complex. From our calculations (PM6//MOZYME in vacuum), we estimate the wild type (WT) barrier to be $7.5 \mathrm{kcal} / \mathrm{mol}$.

Experimentally, specific activity of hydrolysis is determined. Given first order kinetics, saturation of the enzyme with substrate (usual for industrial application) and fast binding and product release, the catalytic rate constant $k_{c a t}$ is directly proportional to the specific activity under the assumption that the amount of active enzyme remains constant. This therefore allows the catalytic rate constant $k_{\text {cat }}$ and, hence, the barrier height to be compared to the improvement factors reported in the results section. The approximations used here in relating the barrier height on the potential energy surface to $k_{c a t}$ have been discussed previously [1].

It is noted that using one CPU per interpolation frame on the reaction barrier, the complete barrier of one mutant can be computed with 10 CPUs usually within less than 12 hours of wall clock time (for a molecular model of the size used in this study). Given a set of molecular models of the enzyme, and 100 available CPUs, it is possible to screen around 1000 mutants within one week.

\section{Combination Mutants}

The molecular model of the enzyme and the positions of the point mutations in the enzyme are illustrated in Fig. 2. The point mutations are listed in Table 2. Two sets of mutants are introduced in this section. 
A benchmarking set $S$ and a combinatorial set $L$, the definitions of which are provided in the following. The point mutations are selected based on different design principles. These are either introduction of structural rearrangements in the active site to change the binding site properties of the active site (residues P38, G39, G41, T42, T103) [2], introduction of space to accomodate the substrate (W104, L278, A282, I285, V286), introduction of dipolar interactions between the enzyme and the substrate (A132, A141, I189) [36] or reduction of polarity in the active site (D223). Of course different heuristic considerations will apply for other enzymes when selecting the single mutations for combinatorial study. The mutants of the benchmarking study are collected in a small set $S$ (22 mutants, Table 1). For the combinatorial study, out of the above we select six residues (G39, T103, W104, A141, I189, L278) which, it is assumed, contribute strongest to increased activity and define the mutations at each position as listed in Table 3. Given the position $i$ and the number of mutations at each position $g_{i}$, in general the upper limit for the number of mutants $M$ in a combinatorial study can be calculated by writing a sum term for each type (i.e. "order") of combination mutant, i.e. single, double, ..., such that

$$
M=\underbrace{\sum_{i} g_{i}}_{\substack{\text { Single } \\
(o=1)}}+\underbrace{\sum_{\substack{i, j \\
j>i}} g_{i} \cdot g_{j}}_{\begin{array}{c}
\text { Double } \\
(o=2)
\end{array}}+\underbrace{\sum_{\substack{i, j, k \\
k>j>i}} g_{i} \cdot g_{j} \cdot g_{k}}_{\begin{array}{c}
\text { Triple } \\
(o=3)
\end{array}}+\ldots
$$

where each sum term consists of $\left(\begin{array}{c}N \\ o\end{array}\right)$ individual terms $(N$ and $o$ being the number of positions which can be mutated and the order of the mutant, respectively). By this scheme, considering the mutations listed in Table 3 , hypothetically $424(=13+64+154+193)$ single to four-fold mutants can be constructed. This number is reduced by applying the restriction that out of the 424 hypothetically possible mutants, 0 single, 2 double, 12 triple and 24 four-fold combination mutants including the pair A141N/Q-I189Y are discarded because in the molecular modeling, these side chains could not be allocated spatially in the same mutant. We further note that 15 out of these remaining 386 mutants (Table 3 ) are present also in the benchmarking set $S$ and thus the combinatorial study consists of 371 unique mutants. A detailed documentation of the number of screened residues in the combinatorial study is provided in Table 4 . Prior to analysis, the reaction barriers of the combination mutants are inspected visually and mutants with irregularly shaped barriers, i.e. consisting of multiple peaks of similar height along the reaction coordinate, are discarded. This step is done simply because the calculations yield inconclusive results, 
so the most conservative choice is to consider it a non-promising candidate for a more active variant. Generating plots of the profiles is completely automated and visual inspection can easily be done for hundreds of mutants. Furthermore, out of the mutants with regular reaction barrier shapes, we discard those mutants with barriers $>19.0 \mathrm{kcal} / \mathrm{mol}$ (i.e. the largest calculated barrier from set $S$ ). Following these selection criteria, 61 mutants are discarded because of inconclusive barrier shapes and 47 mutants because the barrier is higher than $19 \mathrm{kcal} / \mathrm{mol}$ (a distribution of reaction barriers is shown in Fig. S1). After these filtering steps, 278 mutants remain in the combinatorial study which we collect in the large set $L$ (out of which 15 are in set $S$ ). An overview on the distribution of reaction barriers for the mutants from set $L$ is provided Fig. S2 of the supporting information.

We note that in set $S$, all barriers appear regular in shape and no mutant contains the A141N/Q and I189Y pair.

\section{Results and Discussion}

\section{Set $S$ : Calibration of the accuracy}

The correspondance of the computed barriers from set $S$ with the experimental assay is shown in Fig. 3. The exact data is reported in Table 1. A scatterplot of calculated reaction barriers is presented in Fig. 4.

We note that in set $S$, the highest experimentally observed activity is around 11 times the wild type activity (G39A-T103G-W104F-L278A, Table 1), while roughly ten mutants show no increased activity. In total, six mutants show 3 -fold or higher wild type activity. In the calculations, only one mutant is observed to have a lower barrier than the wild type (7.3 kcal/mol, G39A-T103G-L278A) and the highest observed barrier is $18.9 \mathrm{kcal} / \mathrm{mol}$ (I189G).

Given the approximations introduced to make the method sufficiently efficient, it is noted that the intent of the method is not a quantitative ranking of the reaction barriers, but to identify promising mutants for, and to eliminate non-promising mutants from, experimental consideration. Therefore only qualitative changes in overall activity are considered, which are represented by the activity factors $(+1 /-1)$.

We categorize the experimentally observed activities and the predicted reaction barriers as follows. From experiment, a mutant with activity of $1.2(0.8)$ times the wild type activity or higher (lower) is considered 
as improving (degrading). Correspondingly, the computed difference in reaction barrier height between a mutant and the wild type is expressed in qualitative terms. For the comparison with the experimental activity assay, we define a barrier cutoff $\mathrm{c}_{S}=12.5 \mathrm{kcal} / \mathrm{mol}$ to distinguish between potentially improving and degrading mutants in set $S$. The value of $12.5 \mathrm{kcal} / \mathrm{mol}$ is chosen such as to maximize the agreement with experiment, which is $68 \%$, i.e. using a smaller or larger value for the cutoff will decrease this value. A mutant with a predicted barrier $\geq \mathrm{c}_{S}(12.5 \mathrm{kcal} / \mathrm{mol})$ is considered to likely have decreased activity compared to the wild type while mutants with reaction barriers $<\mathrm{c}_{S}$ are considered likely having increased activity.

We note that defining the cutoff is done purely for a post hoc comparison of experimental and computed data. When using the computed barriers to identify promising experimental mutants, one simply chooses the $N$ mutants with the lowest barriers, where $N$ is the number of mutants affordable to do experimentally (e.g. 20 in the discussion of set $L$ ).

Based on this approach, qualitative activity of 15 out of 22 mutants is correctly predicted. It is noted that the correlation is best for mutants with largest activity difference compared to wild type (both positive or negative). For example the method identifies four of the six most active mutants with $\geq 3$-fold wild type activity. Similarly, the method identifies seven out of the eight least active mutants with $\leq 0.5$-fold wild type activity. For mutants with only small differences in activity compared to wild type, the predictions are less accurate.

\section{Set $L$ : Large scale screening study}

Set $L$ is screened to identify new mutants for which increased activity is predicted. The 20 mutants with the lowest barriers are suggested as candidates for further experimental study in Table 5 . The distributions of reaction barriers, resolved by mutations at positions 104 and 189, are shown in Figs. 5A and B. In set $L$, three new mutants are identified with barriers lower than the predicted wild type barrier. Out of the 20 mutants suggested in Table 5, three are double mutants, seven are three-fold and ten are four-fold mutants. No single mutants where found for which increased activity compared to wild type is predicted. All mutants except one contain the G39A mutation, five contain the T103G mutation, six contain a mutation of W104, 13 contain a mutation of A141, 16 contain a mutation of I189 and eight contain the L278A mutation. From this observation it is likely that mutations of G39, A141 and I189 will likely contribute to an increased activity of the mutant and should thus be included in future experimental activity assays. 
Set $L$ is further analysed in terms of the effect of the mutations at the positions 104 and 189. For the mutations of W104, we note that single mutations which give rise to relatively high barriers (W104Q, W104Y, Fig. 5A) can have significantly lower barriers in combination with other mutations. For example, out of the sixty mutants with lowest barriers (Fig. S3), 33 contain a mutation of W104 out of which 17 are suggested to be W104F, while 14 are suggested to be W104Y (two contain W104Q).

The mutation of I189 is analysed in a similar way. In set $L$, five different mutations of this residue are screened (Table 3). The single mutant with the lowest barrier is I189Y and the two mutants with the lowest predicted barrier contain this mutation as well (Table 5). Similarly to above, higher order mutants containing I189A, I189G, I189H or I189N are predicted to have considerably lower barriers than the corresponding single mutants, Fig. 5B. Particularly, out of the mutants listed in Table 5, three contain the I189A, one contains I189G mutation, four contain the I189H mutation and three contain the I189N mutation.

As a special case we highlight that the single mutant I189G has one of the highest calculated barriers (18.9 kcal/mol, Table 1), however the four-fold mutant G39A-A141Q-I189G-L278A has one of the lowest barriers $(6.3 \mathrm{kcal} / \mathrm{mol}$, Table 5). Interestingly, the mutant G39A-A141Q-L278A has an intermediate barrier $(10.9 \mathrm{kcal} / \mathrm{mol})$. It would appear that I189G as a single mutant is counterproductive (high computed barrier) but lowers the barrier of G39A-A141Q-L278A. This observation is further supported by the observation that the I189G mutation is in spatial proximity to A141Q. While it is difficult to quantify the interaction, it is likely that in the mutant, the rather large side chain of A141Q is better accommodated in the active site and can better interact with the substrate.

Observations as these should be kept in mind when selecting the single mutants to be considered when preparing higher order mutants.

\section{Conclusions}

Our previously presented method for high throughput computational screening of mutant activity [1] is benchmarked against experimentally measured amidase activity for 22 mutants of Candida antarctica lipase B (CalB). 
Experimentally, amidase activity is successfully introduced in 12 mutants, the highest activity is determined to be 11.2 -fold over the wild type activity.

Using an appropriate cutoff criterion for the computed barriers, the qualitative activity of 15 out of 22 mutants is correctly predicted. It is noted that the correlation is best for mutants with largest activity difference compared to wild type (both positive and negative). For example the method identifies four of the six most active mutants with $\geq 3$-fold wild type activity. Similarly, the method identifies seven out of the eight least active mutants with $\leq 0.5$-fold wild type activity.

Thus validated, the computational method is used to screen all sterically possible (386) double-, tripleand quadrupole-mutants constructed from the most active single mutants. Based on the benchmark test at least 20 new promising mutants are identified.

These mutants have so far not been tested experimentally and are thus offered as scientifically testable predictions. Interestingly, we observe that single mutants that are predicted to have low activity appear to have high activity in combination with other mutants. This is illustrated in specific analysis of effects of mutations of two different positions (104 and 189).

\section{References}

1. Hediger MR, De Vico L, Svendsen A, Besenmatter W, Jensen JH (2012) A computational methodology to screen activities of enzyme variants. PLoS ONE 7: e49849.

2. Patkar S, Svendsen A, Kirk O, Clausen I, Borch K (1997) Effect of mutation in non-consensus sequence Thr-X-Ser-X-Gly of Candida antarctica lipase B on lipase specificity, specific activity and thermostability. Journal of Molecular Catalysis B: Enzymatic 3: 51-54.

3. Kolkenbrock S, Parschat K, Beermann B, Hinz H, Fetzner S (2006) N-Acetylanthranilate amidase from Arthrobacter nitroguajacolicus Rü61a, an $\alpha / \beta$-hydrolase-fold protein active towards arylacylamides and-esters, and properties of its cysteine-deficient variant. Journal of bacteriology 188: 8430-8440.

4. Nakagawa Y, Hasegawa A, Hiratake J, Sakata K (2007) Engineering of Pseudomonas aeruginosa lipase by directed evolution for enhanced amidase activity: mechanistic implication for amide hydrolysis by serine hydrolases. Protein Engineering Design and Selection 20: 339-346. 
5. Naik S, Basu A, Saikia R, Madan B, Paul P, et al. (2010) Lipases for use in industrial biocatalysis: Specificity of selected structural groups of lipases. Journal of Molecular Catalysis B: Enzymatic 65: $18-23$.

6. Takwa M, Larsen MW, Hult K, Martinelle M (2011) Rational redesign of Candida antarctica lipase B for the ring opening polymerization of d,d-lactide. Chem Commun 47: 7392-7394.

7. Jäckel C, Kast P, Hilvert D (2008) Protein design by directed evolution. Annu Rev Biophys 37: $153-173$.

8. Frushicheva MP, Warshel A (2012) Towards quantitative computer-aided studies of enzymatic enantioselectivity: The case of candida antarctica lipase a. ChemBioChem 13: 215-223.

9. Chica R, Doucet N, Pelletier J (2005) Semi-rational approaches to engineering enzyme activity: combining the benefits of directed evolution and rational design. Current opinion in biotechnology 16: $378-384$.

10. Zanghellini A, Jiang L, Wollacott AM, Cheng G, Meiler J, et al. (2006) New algorithms and an in silico benchmark for computational enzyme design. Protein Science 15: 2785-2794.

11. Zhou T, Caflisch A (2010) High-Throughput Virtual Screening Using Quantum Mechanical Probes: Discovery of Selective Kinase Inhibitors. ChemMedChem 5: 1007-1014.

12. Privett HK, Kiss G, Lee TM, Blomberg R, Chica RA, et al. (2012) Iterative approach to computational enzyme design. Proceedings of the National Academy of Sciences 109: 3790-3795.

13. Suplatov D, Besenmatter W, Švedas V, Svendsen A (2012) Bioinformatic analysis of alpha/betahydrolase fold enzymes reveals subfamily-specific positions responsible for discrimination of amidase and lipase activities. Protein Engineering Design and Selection 25: 689-697.

14. Ishida T, Kato S (2004) Role of Asp102 in the catalytic relay system of serine proteases: a theoretical study. Journal of the American Chemical Society 126: 7111-7118.

15. Noodleman L, Lovell T, Han W, Li J, Himo F (2004) Quantum chemical studies of intermediates and reaction pathways in selected enzymes and catalytic synthetic systems. Chemical reviews 104: 459-508. 
16. Friesner R, Guallar V (2005) Ab initio quantum chemical and mixed quantum mechanics/molecular mechanics (QM/MM) methods for studying enzymatic catalysis. Annu Rev Phys Chem 56: 389427.

17. Rod T, Ryde U (2005) Quantum mechanical free energy barrier for an enzymatic reaction. Physical review letters 94: 138302 .

18. Claeyssens F, Harvey J, Manby F, Mata R, Mulholland A, et al. (2006) High-Accuracy Computation of Reaction Barriers in Enzymes. Angewandte Chemie 118: 7010-7013.

19. Hermann J, Pradon J, Harvey J, Mulholland A (2009) High Level QM/MM Modeling of the Formation of the Tetrahedral Intermediate in the Acylation of Wild Type and K73A Mutant TEM-1 Class A $\beta$-Lactamase. The Journal of Physical Chemistry A 113: 11984-11994.

20. Tian L, Friesner R (2009) Qm/mm simulation on p450 bm3 enzyme catalysis mechanism. Journal of chemical theory and computation 5: 1421-1431.

21. Parks J, Hu H, Rudolph J, Yang W (2009) Mechanism of Cdc25B phosphatase with the small molecule substrate p-nitrophenyl phosphate from QM/MM-MFEP calculations. The Journal of Physical Chemistry B 113: 5217-5224.

22. Altarsha M, Benighaus T, Kumar D, Thiel W (2010) Coupling and uncoupling mechanisms in the methoxythreonine mutant of cytochrome P450cam: a quantum mechanical/molecular mechanical study. Journal of Biological Inorganic Chemistry 15: 361-372.

23. Himo F (2006) Quantum chemical modeling of enzyme active sites and reaction mechanisms. Theoretical Chemistry Accounts: Theory, Computation, and Modeling (Theoretica Chimica Acta) 116: $232-240$.

24. Hu L, Eliasson J, Heimdal J, Ryde U (2009) Do Quantum Mechanical Energies Calculated for Small Models of Protein-Active Sites Converge? The Journal of Physical Chemistry A 113: 11793-11800.

25. Ishida T, Kato S (2003) Theoretical perspectives on the reaction mechanism of serine proteases: the reaction free energy profiles of the acylation process. Journal of the American Chemical Society 125: $12035-12048$. 
26. Hedstrom L, et al. (2002) Serine protease mechanism and specificity. Chemical reviews 102: 45014524.

27. Fersht A (1985) Enzyme Structure and Mechanism. W.H. Freeman and Company: CRC Press, Inc.

28. Polgár L (1989) Mechanisms of protease action. CRC Press, Inc.

29. Henke E, Bornscheuer U (2003) Fluorophoric assay for the high-throughput determination of amidase activity. Analytical chemistry 75: 255-260.

30. Gao J, Truhlar DG (2002) Quantum mechanical methods for enzyme kinetics. Annual Review of Physical Chemistry 53: 467-505.

31. Stewart J (1996) Application of localized molecular orbitals to the solution of semiempirical selfconsistent field equations. International Journal of Quantum Chemistry 58: 133-146.

32. Stewart J (2007) Optimization of parameters for semiempirical methods V: Modification of NDDO approximations and application to 70 elements. Journal of Molecular Modeling 13: 1173-1213.

33. Stewart J (1990) Mopac: a semiempirical molecular orbital program. Journal of Computer-Aided Molecular Design 4: 1-103.

34. Stewart J (2009). Mopac2009. HTTP://OpenMOPAC.net.

35. Uppenberg J, Oehrner N, Norin M, Hult K, Kleywegt G, et al. (1995) Crystallographic and molecular-modeling studies of lipase B from Candida antarctica reveal a stereospecificity pocket for secondary alcohols. Biochemistry 34: 16838-16851.

36. Syrén PO, Hendil-Forssell P, Aumailley L, Besenmatter W, Gounine F, et al. (2012) Esterases with an Introduced Amidase-Like Hydrogen Bond in the Transition State Have Increased Amidase Specificity. ChemBioChem 13: 645-648. 


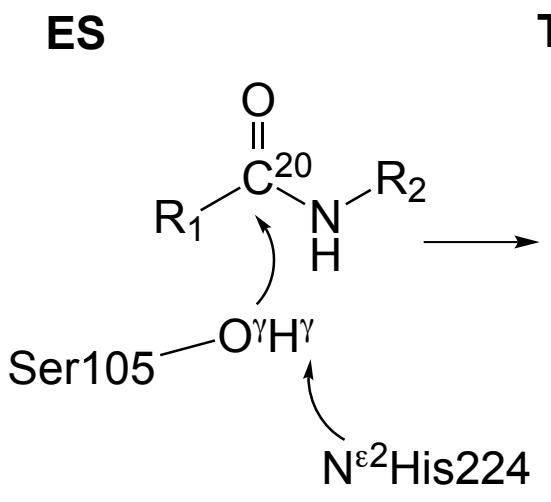

TS

TI
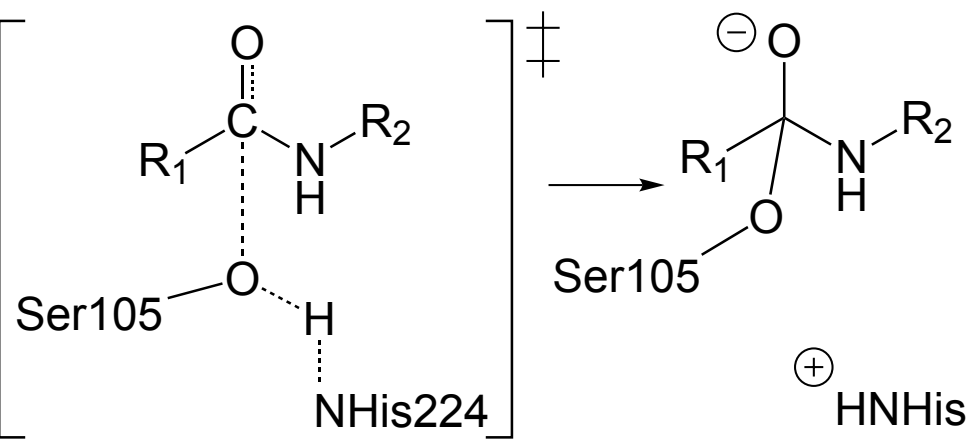

Figure 1. Reaction scheme for the formation of TI. Nucleophilic attack by $\mathrm{O}^{\gamma}$ of $\mathrm{S} 105$ on carbonyl carbon $\mathrm{C}^{20}$ of substrate. $\mathrm{R}_{1}$ : $-\mathrm{CH}_{2}-\mathrm{Cl}, \mathrm{R}_{2}$ : $-\mathrm{CH}_{2}-\mathrm{C}_{5} \mathrm{H}_{6}$. 
A
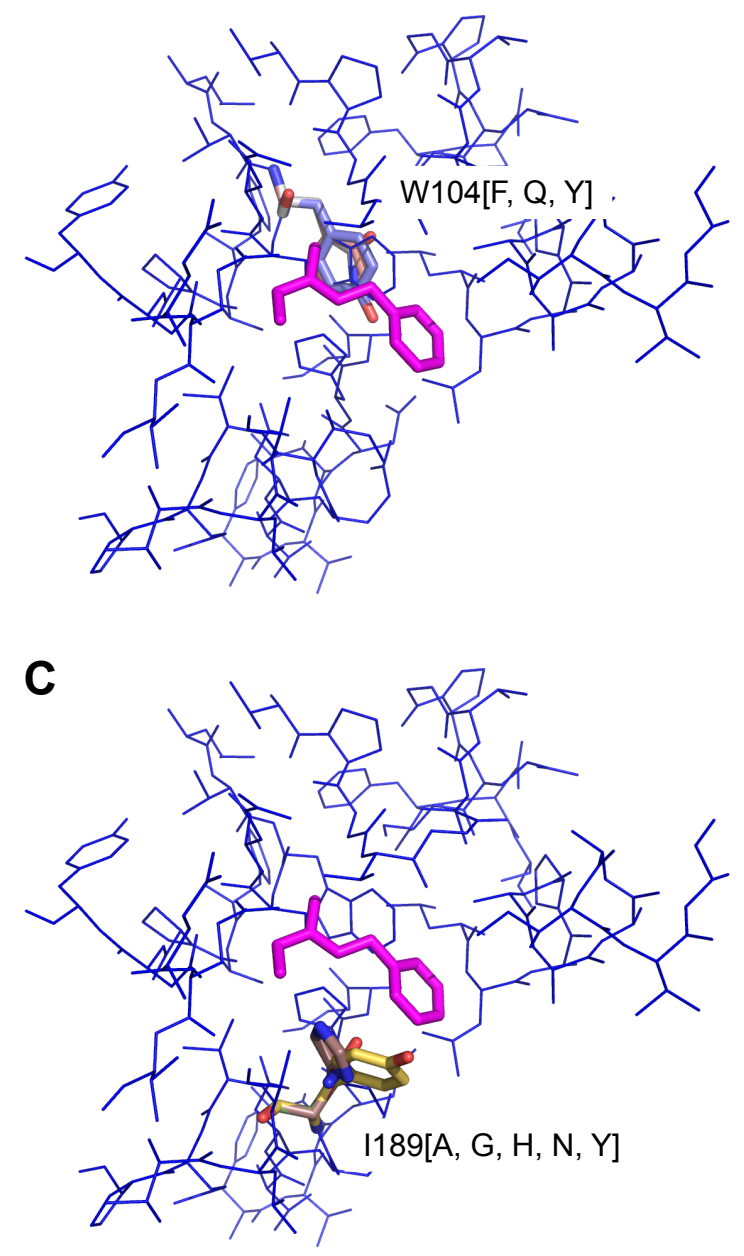

B
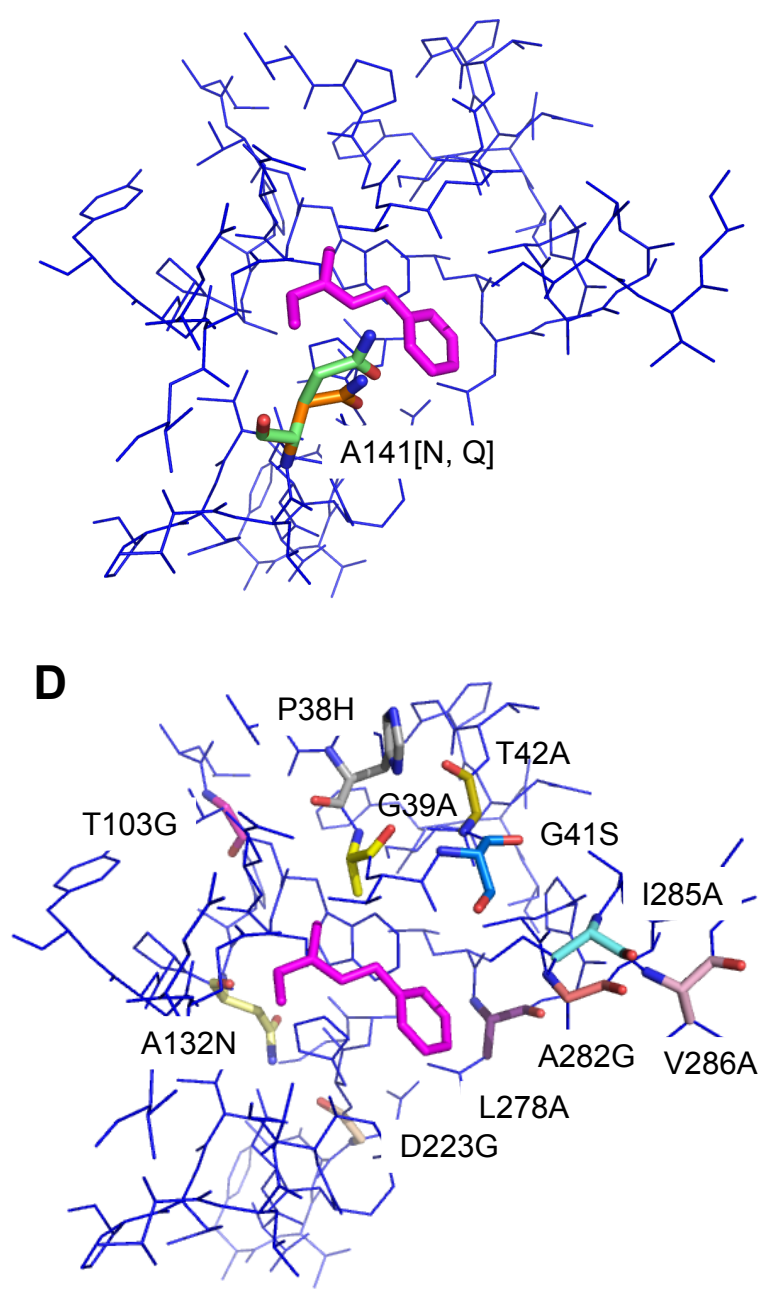

Figure 2. Positions of point mutations. A: Overlay of mutations W104[F, Q, Y]. B: Overlay of mutations of A141[N, Q]. C: Overlay of mutations of I189[A, G, H, N, Y]. D: Mutations P38H, G39A, G41S, T42A, T103G, A132N, L278A, A282G, I285A and V286A. Substrate shown in magenta. 


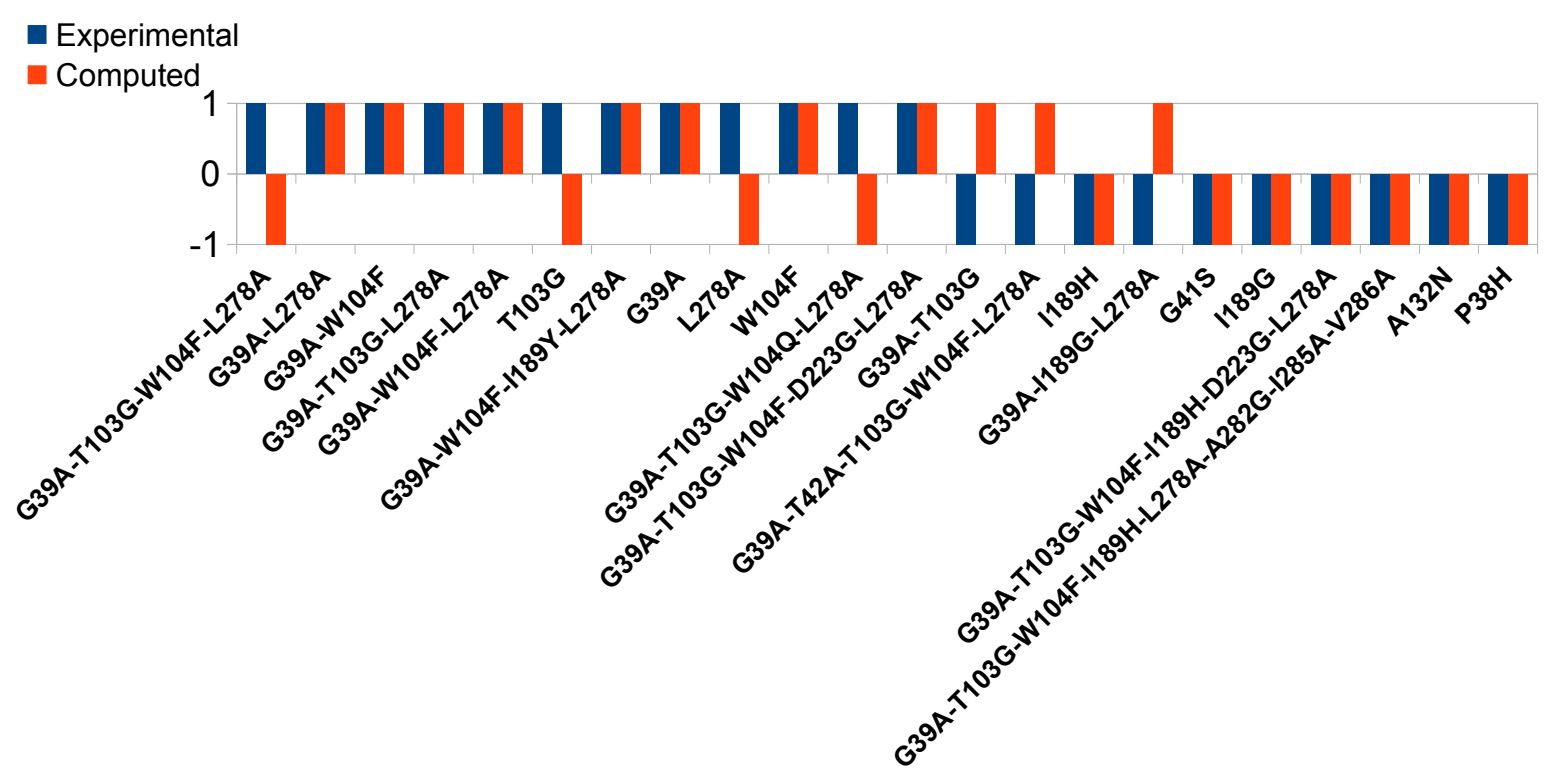

Figure 3. Comparison of experimental and computed activities. $1 /-1$ correspond to increased/decreased overall activity, respectively. Prediction rate is 15/22 (68\%). 
PM6//MOZYME

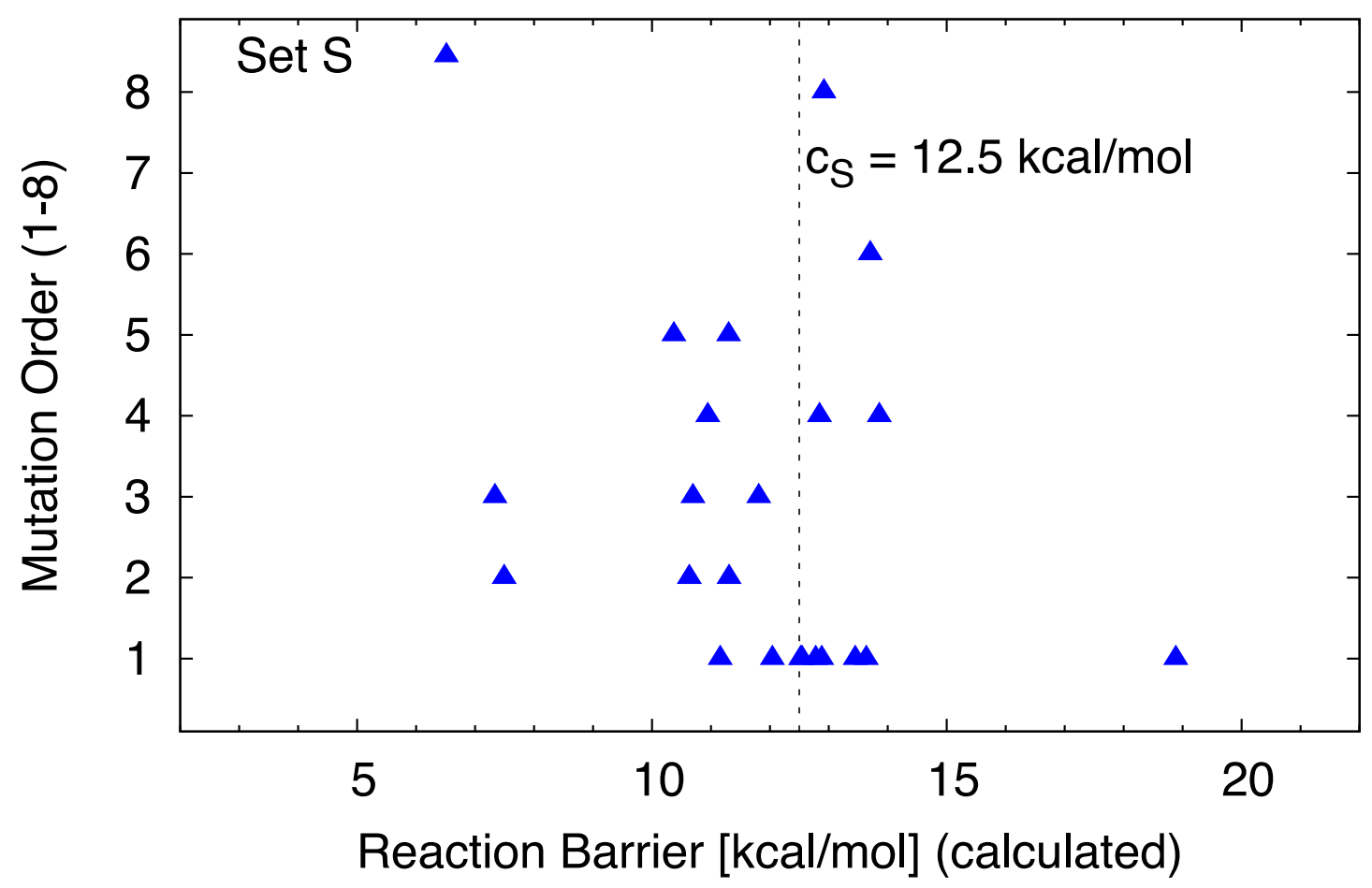

Figure 4. Barrier scatter plot of set $S .22$ mutants; The cutoff value $\mathrm{c}_{S}$ is discussed in the text. 

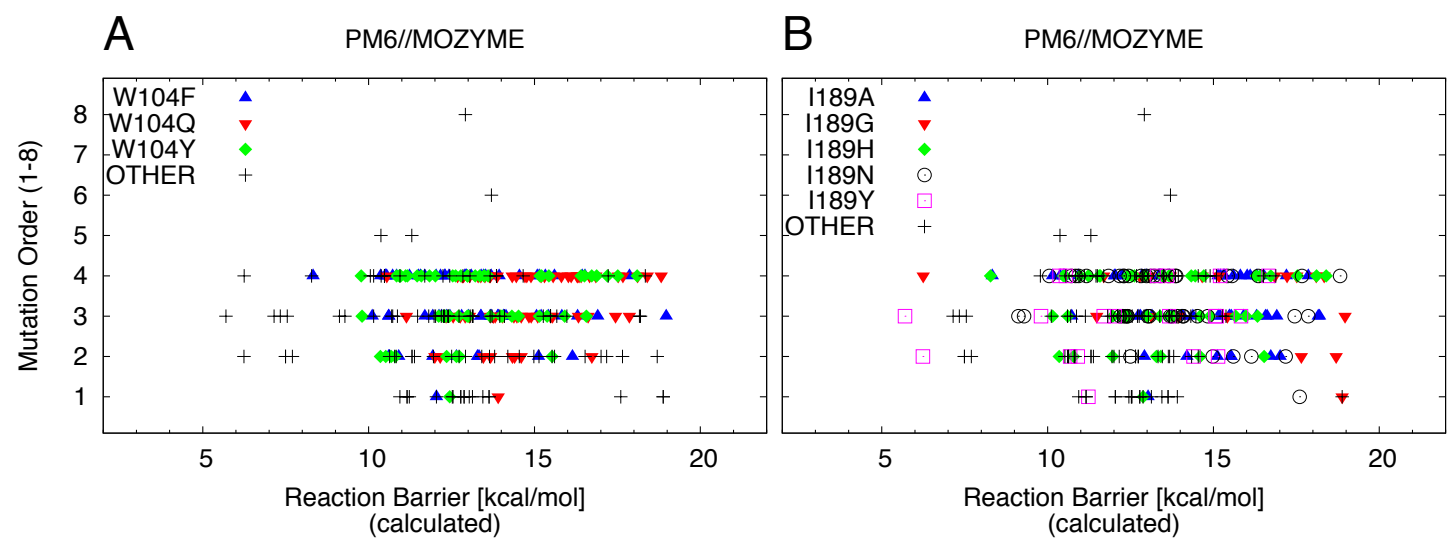

Figure 5. Barrier scatter plots of set $L$. In both panels, the labels indicate mutants containing the labeled and possibly additional mutations up to the indicated order. "OTHER" indicates a mutant not containing any of the labeled mutations or of higher than 4. order. A: Mutations of W104. B:

Mutations of I189. 


\section{Tables}

Table 1. Experimental overall activities and calculated reaction barriers of Set $S$. Activity factors $+1 /-1$ indicate increased/decreased overall activity. $A o$ and $P p$ indicating expression in organisms (Org.) Aspergillus oryzae or Pichia pastoris, respectively. The cutoff to distinguish higher and lower activity mutants is defined as $12.5 \mathrm{kcal} / \mathrm{mol}$, see text.

\begin{tabular}{|c|c|c|c|c|c|}
\hline \multirow[t]{2}{*}{ Species } & \multicolumn{2}{|c|}{ Experimental } & \multicolumn{2}{|c|}{ Calculated } & \multirow[b]{2}{*}{ Org. } \\
\hline & $\begin{array}{l}\text { Activity } \\
{[* \mathrm{WT}]}\end{array}$ & $\begin{array}{l}\text { Activity- } \\
\text { Factor }\end{array}$ & $\begin{array}{l}\text { Barriers } \\
\text { [kcal/mol] }\end{array}$ & $\begin{array}{l}\text { Activity- } \\
\text { Factor }\end{array}$ & \\
\hline G39A-T103G-W104F-L278A & 11.2 & 1 & 13.9 & -1 & Ao \\
\hline G39A-L278A & 7.0 & 1 & 11.3 & 1 & $P p$ \\
\hline G39A-W104F & 4.2 & 1 & 10.6 & 1 & Ao \\
\hline G39A-T103G-L278A & 3.8 & 1 & 7.3 & 1 & Ao \\
\hline G39A-W104F-L278A & 3.6 & 1 & 11.8 & 1 & $P p$ \\
\hline T103G & 3.0 & 1 & 13.6 & -1 & Ao \\
\hline G39A-W104F-I189Y-L278A & 2.9 & 1 & 10.9 & 1 & $P p$ \\
\hline G39A & 2.8 & 1 & 11.2 & 1 & Ao \\
\hline L278A & 2.5 & 1 & 12.8 & -1 & Ao \\
\hline W104F & 2.0 & 1 & 12.0 & 1 & Ao \\
\hline G39A-T103G-W104Q-L278A & 1.9 & 1 & 12.8 & -1 & Ao \\
\hline G39A-T103G-W104F-D223G-L278A & 1.5 & 1 & 11.3 & 1 & $P p$ \\
\hline G39A-T103G & 0.8 & -1 & 7.5 & 1 & Ao \\
\hline G39A-T42A-T103G-W104F-L278A & 0.7 & -1 & 10.4 & 1 & $P p$ \\
\hline $\mathrm{I} 189 \mathrm{H}$ & 0.5 & -1 & 12.9 & -1 & $P p$ \\
\hline G39A-I189G-L278A & 0.4 & -1 & 10.7 & 1 & $P p$ \\
\hline G41S & 0.3 & -1 & 13.4 & -1 & $P p$ \\
\hline I189G & 0.2 & -1 & 18.9 & -1 & $P p$ \\
\hline G39A-T103G-W104F-I189H-D223G-L278A & 0.1 & -1 & 13.7 & -1 & $P p$ \\
\hline G39A-T103G-W104F-I189H-L278A-A282G-I285A-V286A & 0.1 & -1 & 12.9 & -1 & $P p$ \\
\hline A132N & 0.0 & -1 & 12.5 & -1 & $P p$ \\
\hline P38H & 0.0 & -1 & 12.5 & -1 & $P p$ \\
\hline WT & 1.0 & -- & 7.5 & -- & \\
\hline
\end{tabular}


Table 2. Point mutations. The term active site refers to residues with potential direct Van der Waals contact to the substrate. The term first shell/second shell refers to residues which are adjecent to an active site/first shell residue.

\begin{tabular}{rlll} 
Target & Mutations & Type & Description \\
\hline P38 & H & Second shell & (H neutral) \\
G39 & A & First shell & \\
G41 & S & First shell & \\
T42 & A & Second shell & \\
T103 & G & First shell & \\
W104 & F, Q, Y & Active site & \\
A132 & N & First shell & \\
A141 & N, Q & Active site & (G including additional water, \\
I189 & A, G, H, N, Y & Active site & H neutral) \\
& & First shell & (Increase of charge by +1) \\
D223 & G & Active site & \\
L278 & A & Active site & \\
A282 & G & Active site & \\
I285 & A & First shell & \\
V286 & A & &
\end{tabular}


Table 3. Side chains used for generation of combinatorial set $L . i$ and $g_{i}$ indicate the position in the back bone and the number of mutations at that position, respectively.

\begin{tabular}{lrc} 
Mutation & $i$ & $g_{i}$ \\
\hline G39A & 39 & 1 \\
T103G & 103 & 1 \\
W104\{F, Q, Y $\}$ & 104 & 3 \\
A141 $\{$ N, Q & 141 & 2 \\
I189 $\{$ A, G, H, N, Y $\}$ & 189 & 5 \\
L278A & 278 & 1 \\
\hline
\end{tabular}


Table 4. Combinatorial study details. From the possible mutants, the combinations containing the pair A141N/Q-I189Y, the mutants with inconclusive barriers and the mutants with barriers $>19.0 \mathrm{kcal} / \mathrm{mol}$ are subtracted to give the number of mutants in set $L$. "Only Set L" indicates the number of mutants uniquely present in set $L$ and not in set $S$.

\begin{tabular}{lr|rrrr|rr} 
Order & Possible & \multicolumn{1}{c}{$\begin{array}{c}\text { Containing } \\
\text { A141N/Q-I189y }\end{array}$} & $\begin{array}{l}\text { Inconclusive } \\
\text { barrier }\end{array}$ & $\begin{array}{l}\text { Barrier }>19.0 \\
\text { [kcal/mol] }\end{array}$ & Set $L$ & \multicolumn{2}{c}{ Only } \\
Set $L$
\end{tabular}


Table 5. Selection of mutants from set $L$ with lowest barriers.

\begin{tabular}{lr} 
Mutation & Barrier [kcal/mol] \\
\hline G39A-T103G-I189Y & 5.7 \\
G39A-I189Y & 6.2 \\
G39A-A141Q-I189G-L278A & 6.3 \\
G39A-A141N-L278A & 7.6 \\
G39A-A141N & 7.7 \\
G39A-A141N-I189H-L278A & 8.3 \\
G39A-W104F-A141Q-I189A & 8.3 \\
G39A-A141Q-I189N & 9.1 \\
G39A-A141N-I189N & 9.3 \\
G39A-T103G-W104Y-A141N & 9.8 \\
G39A-W104Y-I189Y & 9.8 \\
G39A-A141N-I189N-L278A & 10.1 \\
G39A-W104F-A141N & 10.1 \\
G39A-I189H-L278A & 10.2 \\
G39A-A141N-I189A-L278A & 10.2 \\
W104Y-I189H & 10.4 \\
G39A-T103G-W104F-I189Y & 10.4 \\
G39A-A141Q-I189A-L278A & 10.4 \\
G39A-T103G-A141Q-I189H & 10.4 \\
G39A-T103G-I189A-L278A & 10.5 \\
\hline
\end{tabular}




\section{Figure 1}

Reaction scheme for the formation of TI.

Nucleophilic attack by $\mathrm{O}^{\mathrm{\gamma}}$ of $\mathrm{S} 105$ on carbonyl carbon $\mathrm{C}^{20}$ of substrate. $\mathrm{R}_{1}:-\mathrm{CH}_{2}-\mathrm{Cl}, \mathrm{R}_{2}:-\mathrm{CH}_{2}-\mathrm{C}_{5} \mathrm{H}_{6}$.

ES

TS

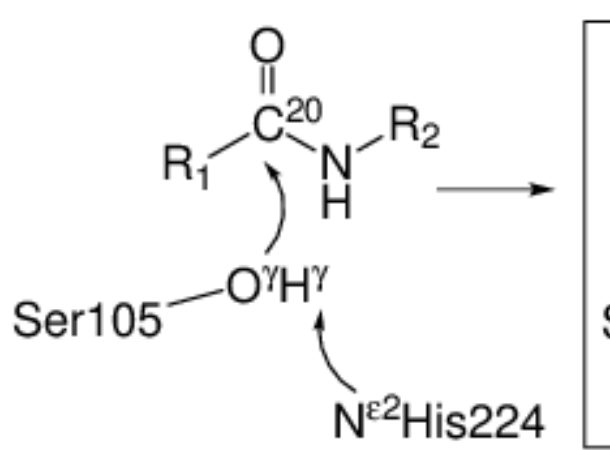

TI<smiles>[R20]NC([R])(N[R6])O[R5]</smiles><smiles>[R2]NC([R])([O-])O[AsH2]</smiles>

$\oplus$ $\mathrm{HNHis}$ 


\section{Figure 2}

Position of point mutations.

A. Overlay of mutations W104[F, Q, Y]. B: Overlay of mutations A141[N, Q]. C: Overlay of mutations of I189[A, G, H, N, Y]. D: Mutations P38H, G39A, G41S, T42A, T103G, A132N, L278A, A282G, I285A, V286A. Substrate shown in magenta.

A

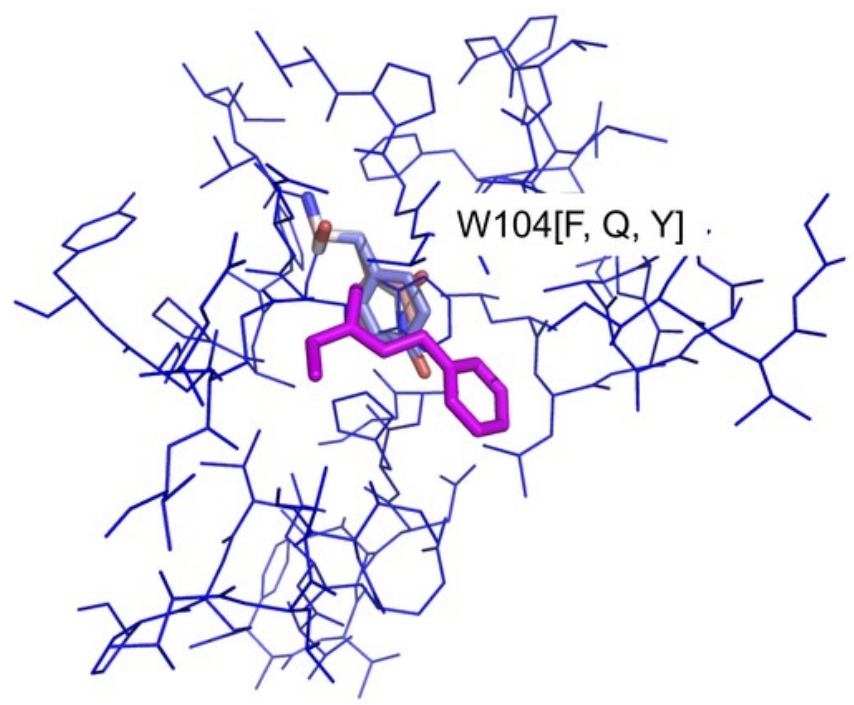

C

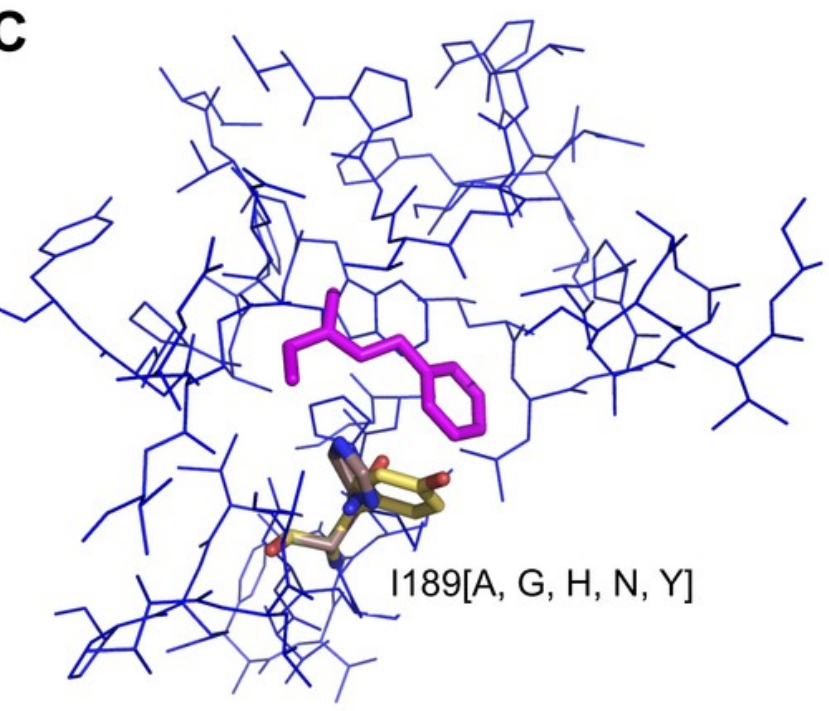

B

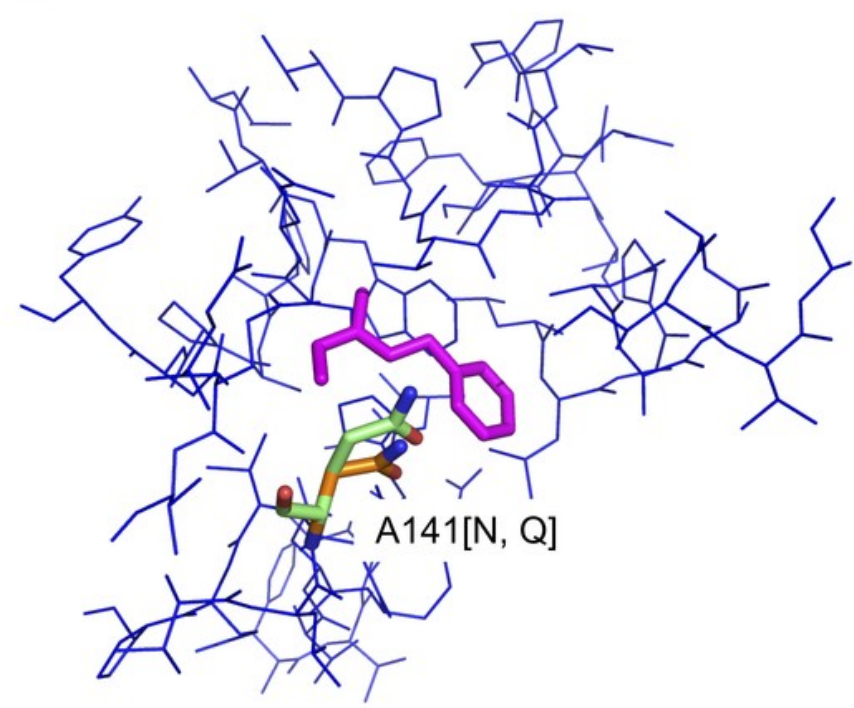

D

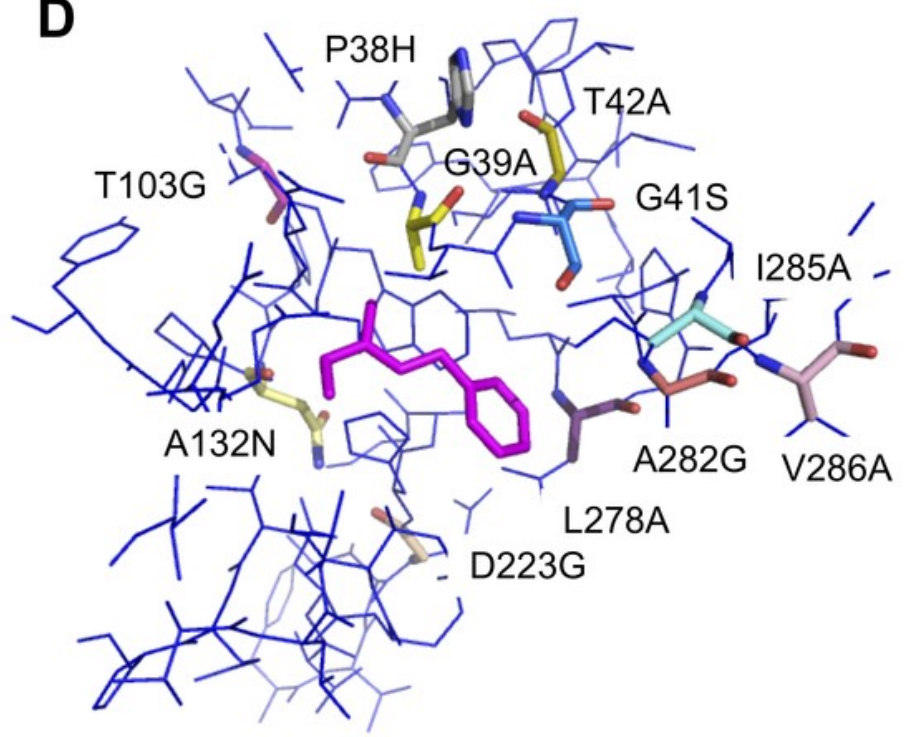




\section{Figure 3}

Comparison of experimental and computed activities.

1/-1 correspond to increased/decreased overall activity, respectively. Prediction rate is 15/22 (68\%).

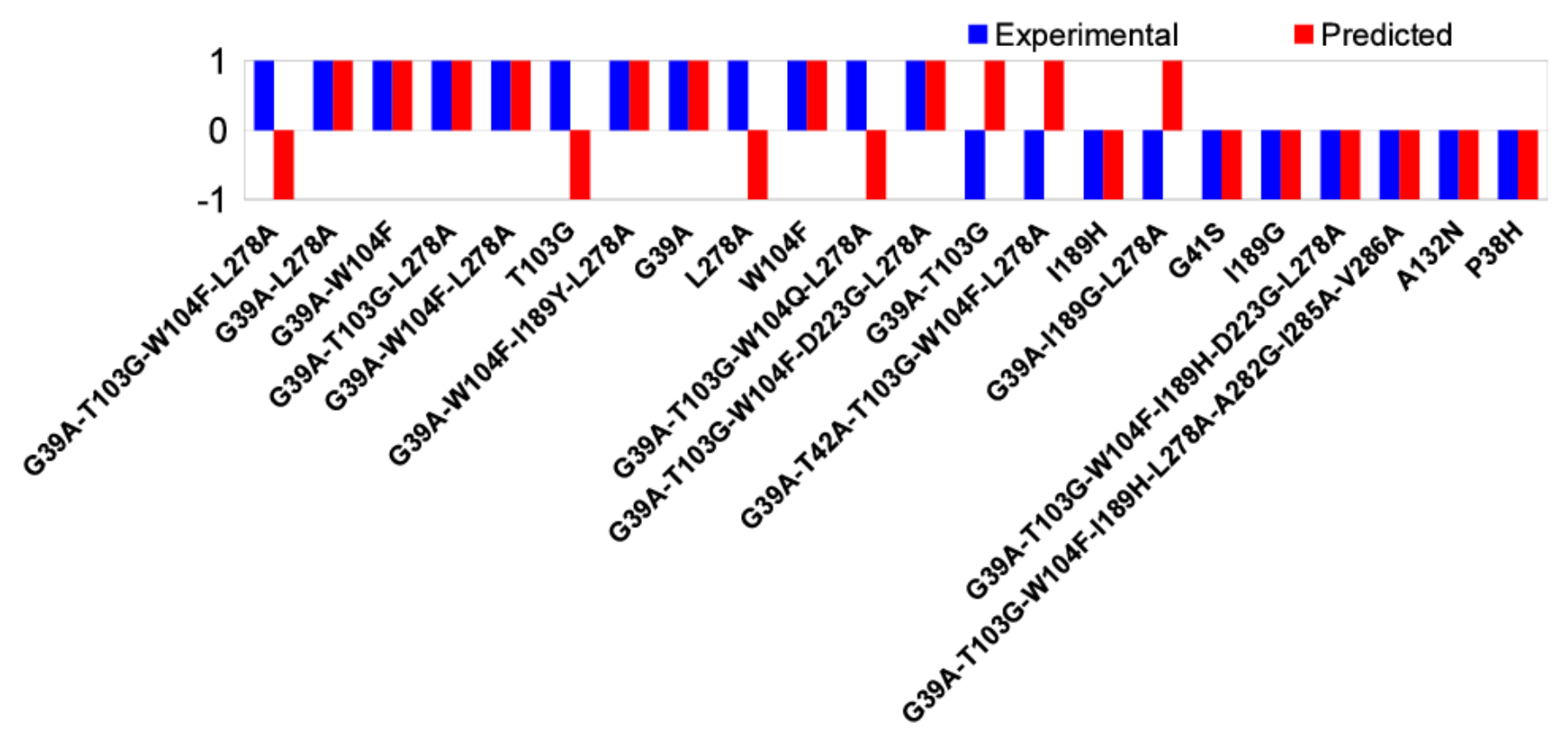




\section{Figure 4}

\section{Barrier scatter plot of set $S$.}

22 mutants; The cutoff value $\mathrm{c}_{S}$ is discussed in the text.

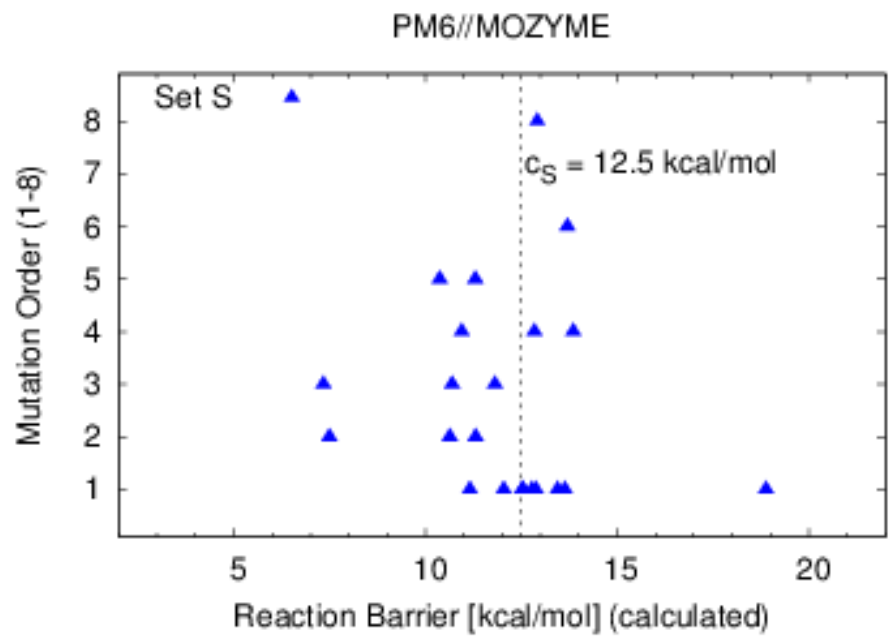




\section{Figure 5}

Barrier scatter plots of set $L$.

In both panels, the labels indicate mutants containing the labeled and possibly additional mutations up to the indicated order. "OTHER" indicates a mutant not containing any of the labeled mutations or of higher than 4. order. A: Mutations of W104. B: Mutations of I189.
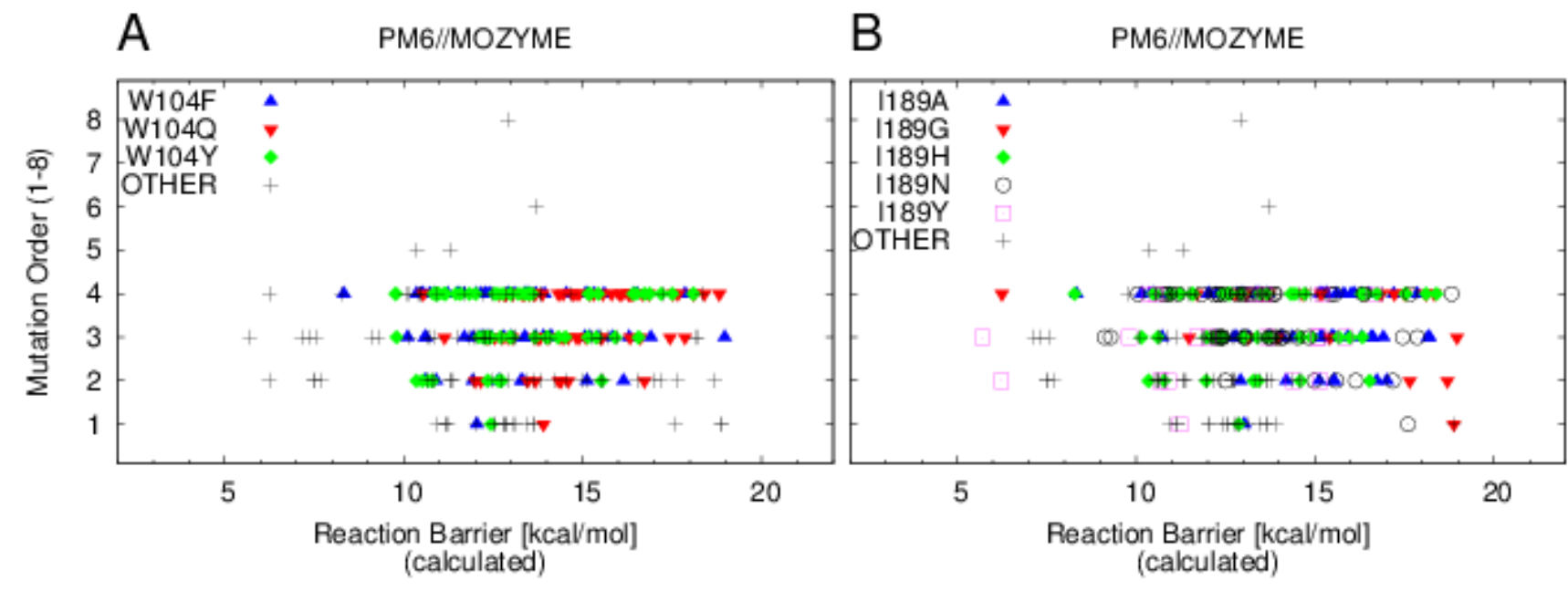